www.jmscr.igmpublication.org

Impact Factor (SJIF): 6.379

Index Copernicus Value: 79.54

ISSN (e)-2347-176x ISSN (p) 2455-0450

crossrefDOI: https://dx.doi.org/10.18535/jmscr/v6i11.90

\title{
Seroconversion and Prevalence of TTIs (Transfusion Transmissible Infections) among Blood Donors in Southern Part of India
}

\section{Authors \\ Kodandarao Kuna ${ }^{1}$, Padmanabham Yalangi ${ }^{2}$, Hemaprakash Pilli $^{3}$ Lakshmipathy Chityala ${ }^{4}$}

${ }^{1}$ Department of General Surgery, GITAM Institute of Medical Sciences and Research, Visakhapatnam

${ }^{2,3}$ Department of Microbiology, GITAM Institute of Medical Sciences and Research, Visakhapatnam

${ }^{4}$ Incharge of APSACS \& ART Centre, District Hospital, Rajamehendravaram, India

Corresponding Author

Padmanabham Yalangi

Associate Professor, Dept. of Microbiology, GIMSR (Gitam Institute of Medical Sciences and Research),

Visakhapatnam, India

Email: kkraomsgs@gmail.com

\begin{abstract}
Aims \& Objectives: Effective screening of donors blood as per WHO guidelines and to assess the sero prevalence of HIV, HBsAg, HCV, Syphilis and Malaria among population in a locality in southern part of India based on the data of screened donor blood bags.

Material \& Methods: During the period from 2012 to Aug 2018, a total number of 17,635 samples from the donated blood bags from a dedicated blood bank in southern part of India are screened for sero infections as per WHO guidelines using Elisa reader and RPR kit. The emphasis is on seroconversion when the tests result positive. The conclusive data is also compared with similar data in other parts of India studied by other eminent authors.

Results \& Discussion: It is observed that 297 samples are positive for HBsAg (1.68\%), 68 samples are positive for HIV $(0.38 \%)$, and 59 samples are positive for HCV $(0.33 \%)$. Syphilis and Malaria reported not much significant as per Chi-square value. The data on comparision with other similar data showed similar trend except in the large sample study of 2,20,432 where syphilis reported high prevalence.

Conclusion: Strict quality control, counselling of donars, elimination of paid repeated donors, using the latest time tested gadgets to screen the blood, avoiding contamination, training of blood bank lab technicians at regular intervals updating knowledge, maintaining regular uninterrupted power supply and avoiding resource crunch are very very essential for the best possible quality of blood transfusion.

Keywords: Blood, donor, seroconversion, prevalence, screening etc.
\end{abstract}

\section{Introduction}

Safe blood is a universal right. Blood transfusion has been a routine process in almost all hospitals worldwide. It is fairly acceptable by the public. It is thoroughly screened for seropositive infections like hepatitis B \& C, HIV, Syphillis, Malaria commonly and rarely for TORCH infections in some centers. Measuring 
their severity, WHO recommended pretransfusion blood tests for HIV, HBV, HCV and syphilis as mandatory as blood transfusion carries the risk of $\mathrm{TTI}^{20}$ (Transfusion - transmissible infections ). With each unit of blood transfused to the patient there is $1 \%$ chance of TTI to transmit thereby causing significant health risk \& economic burden to the person and the society.

\section{Indian Scenario}

The present trend in blood transfusion in India indicates an increase in TTI. India carries a burden of $50-65$ millions of $\mathrm{HBV}$ carriers and about 3 million HIV cases. Hence TTI can cause grave consequences if not contained soon. In India the overall sero prevalence is $4.1 \%$. India as a population of more than 1.3 billion with $5-7$ million HIV +ve cases, 43 million HBV +ve cases and 15 million $\mathrm{HCV}+\mathrm{ve}$ persons. A very large study of 2,20,482 samples by AKANKSHA RAWAT et al in 2014 from Delhi reported HIV prevalence of $0.32 \%$ and overall seroprevalence of $4.36 \%$. In India the national prevalence is 0.36 $\%$. HIV II, less aggressive is limited to western Africa. With HAART (Highly active anti retro viral therapy ) treatment for HIV, $80 \%$ reduction of mortality is achieved. The HAART drugs act by inhibiting reverse transcriptase and protease synthesis of the HIV viral cell.

\section{Sero Prevalence in Indian Donor Population}

$\begin{array}{llll}\mathrm{HIV} & - & - & 0.3 \% \\ \mathrm{HBV} & - & - & 2-8 \% \\ \mathrm{HCV} & - & - & 2 \%\end{array}$

Indian subcontinent is classified as an intermediate hepatitis " $\mathrm{B}$ " virus endemic ( $\mathrm{HBsAg}$ carriage $2-7 \%$ ) zone. In India $2-5 \%$ of general population is chronically infected with $\mathrm{HBV}$ as per WHO Hep-B fact sheet ${ }^{15}$ 2017. Though malarial parasitemia is low in Indian population except in some hilltop areas during recrudescence, very high prevalence of $30.2 \%$ is reported as per Nigerian teaching hospital study. Mangalore study reports prevalence of MP of $0.01 \%$ in Indian population. Study in Cameroon country by Yaounde et al reports $16.5 \%$ donors positive for plasmodium species.

\section{Epidemiology ${ }^{19}$}

India is the second largest global pool of seroinfections. $60 \%$ of the world's sero-infections exist in developing countries. 98,000 people die each year needlessly due to preventable medical errors. International prevalence of $\mathrm{HCV}$ ranges from $0.42-1.2 \%$. Globally $130-150$ million people have chronic hepatitis ' $\mathrm{C}$ '. There is no vaccine for Hepatitis ' $C$ ' unlike for Hepatitis ' $B$ '. In 2007, WHO estimated 33.2 million PL HIV (people living with HIV) with 2.5 million new infections and 2.1 million deaths. In Africa HIV prevalence exceeds even $15 \%$, Fasola et al reported $13.2 \%$ prevalence of HIV in Nigeria. Cumulative death toll due to HIV/AIDS is 20 million worldwide (11.4 million among sub Saharan children). Between $2001 \& 2007$ there is steepest increase of AIDS cases by $90 \%$ in ASIA. When overall sero-prevalence in USA and Europe is 1 in $5,00,000$ population in developing countries the rate is $5-10 \%$ of blood transfusions. In USA HBV prevalence is 1/2,70,000, and HIV 1/2,35,000 and Europe HBV 1/70,000, HIV 1/1-5 million. Even in remote parts of India like at Jorhat, Assam the prevalence of HBsAg is $0.57 \%$ and $\mathrm{HCV} 0.42 \%$ as per the recent study period of 2013 to 2016. WHO report further states that the viral dose of HIV transmission through blood is so large that $1 \mathrm{HIV}$ +ve transfusion through blood leads to death in children after two years and adults after $3-5$ years.

Transfusion Malaria: In developing countries> 50 cases per million donor units report transfusion malaria. Risk is least in blood bags stored for more than two weeks and high in less than five days. Because malaria parasite is found in red blood cells of an infected person, it can be transmitted through blood transfusion, organ transplant or shared needles/syringes. Merozoites in blood stream adhere to $\mathrm{RBC}$ via specific receptors (in Vivax duffy blood group antigen, $\mathrm{fy}^{\mathrm{a}}$ fy $^{b}$ ) and PfEmp- 1 antigen. P.vivax is most frequently observed in transfusion malaria and also P.malariae, which has long survival time in the human. Transfusion of whole blood are packed 
cell RBC transmit malaria often. It has short incubation period of $2-4$ days, low grade parasitemia, periodicity \& relapses are absent. Gamma irradiation, photochemical \& photodynamic inactivation done in blood banks can minimise transfusion malaria. Engyme immuno assays (EIA), automated protein micro array based tests have good antigen-antibody sensitivity. Antimicrobial agents given to recipients routinely can minimise transfusion malaria burden to a great extent.

\section{Seroconversion}

Seroconversion is defined as the appearance of specific anti HIV/HBV/HCV anti bodies in serum in 8 weeks after exposure. HIV is a single stranded RNA retrovirus. It is spread primarily by sexual exposure and also by blood, needle prics, vertical transmission from mother to child. Primary infection of HIV is symptomatic in $70-$ $80 \%$ of cases, occurs in 2-4 weeks after exposure. $75 \%$ of transmission of HIV is heterosexual, 5 $10 \%$ of new HIV are in children and more than $90 \%$ of them are affected during pregnancy. Mother and child vertical transmission is $25-44$ $\%$ in developing countries. HBV is hepadna DNA virus of $4-20$ weeks incubation period, spread by blood saliva, sexual, syringes and vertical. Vaccine is available for HBV not for HIV and $\mathrm{HCV}$. HCV is a flavivirus-RNA spreads by blood and saliva and not sexual nor vertical.

Among health care workers in developing countries $40 \%$ of HIV infection is due to reused syringes \& needles. With the help of nucleic acid based assays the risk of TTi's can be minimised by diagnosing the infection early and accurately even with minimal viral load which is achieved greatly by USA \& EU. WHO recommended the use of latest generation of Elisa and rapid diagnostic genetic assays. The sero active blood bags have to be discarded as per WHO guidelines.

Safe blood is a universal right is WHO's slogan. With HAART (Highly active anti retro viral therapy) regimen $80 \%$ of patients have undetectable viral load (VL) of < 50 copies per $\mathrm{ml}$ at 4-6 months of treatment from the high VL of $>1,00,000$ copies per $\mathrm{ml}$. Nevirapine \&
Efavirenz drugs are of immense value in controlling HIV viral replication. Entecavir \& Telbivudine achieve HBV - DNA negativity in $90 \%$ of patients at one year of treatment of HBV. Graphs shown below clearly indicate the seroconversion of $\mathrm{HBV}$ and the carrier state and the next graph depicts seroconversion of HIV which are helpful in planning the drug treatment and resistance. Nucleic acid based assays of latest generation based on molecular biology of HIV, HBsAg, HCV are of great value in detecting even the lowest viremia even during window/latent phase of seroconversion. As such genetic based gadgets are used in the west the TTi prevalence rate is the lowest in the world. Only snag with such gadgets is they are not cost effective for developing countries with population explosion.

The molecular structure of Hepatitis ' $\mathrm{B}$ ' virus which is useful in targeting the drug action. HBV mutation and the natural course of Hepatitis ' $B$ ' disease with different phases of seroconversion like tolerance, clearance, latency and mutation which are significant in planning the time of performing the screening and diagnostic test to minimise TTi. Whereas Graph 1 gives a telltale picture of the amplitude of titre of different HBV antibodies which are of immense value in controlling the disease and minimising the spread of it. Graph 2 about the biological behaviour of HIV gives an idea about the seroconversion that takes place during the first few weeks of the total progression of the disease from 4 weeks to 3 years. The full blown picture of AIDS disease develops at the other end of the time scale of HIV. During the course the CD 4 titre falls after initial rise with the viral load (VL) increasing with advancing time. Untreated HIV cases can progress to AIDS in $25-35 \%$ of patients.

Co-Infection ${ }^{17}$ : Co-infection of $\mathrm{HBV}$ with $\mathrm{HCV}$ or HIV can happen and them the natural course of disease is progressive and aggressive. Interferon therapy is effective. Determinents of co-infection are $80 \%$ for haemophilic, $72-80 \%$ for injectable drug users, $3-5 \%$ from heterosexuals and $10 \%$ among MSM. Only $15-20 \%$ clear the infection. Such case finding among donors must be referred 
to the fast track medical committee for immediate treatment. Co-infection are not reported in this study. The study by Fulzele et al from Mumbai in 2016 reported 2 cases among 16,899 donors.

\section{${ }^{22}$ Graph 1}

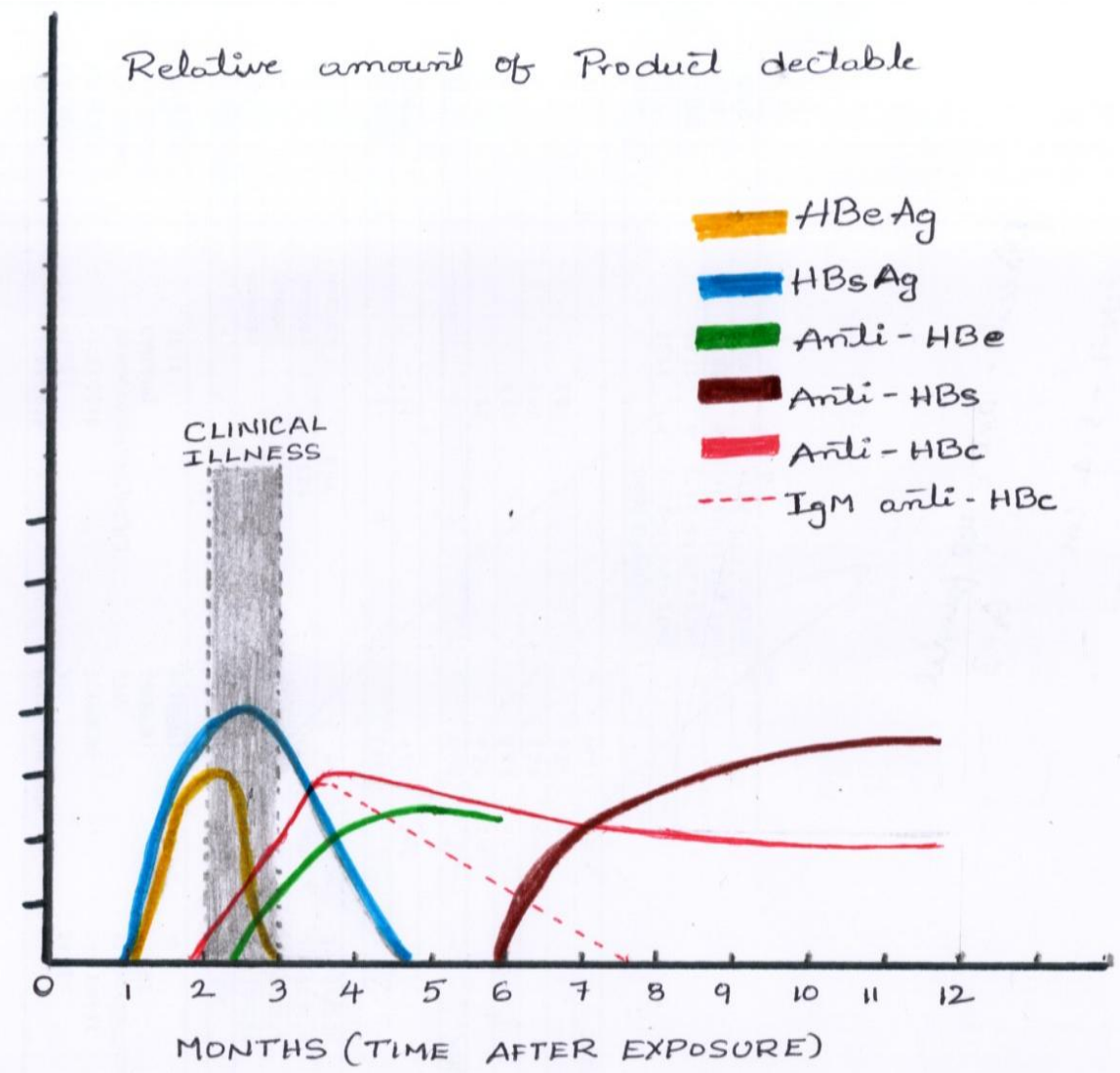

Sero response \& seroconversion of Hepatitis ' $B$ ' virus

\section{${ }^{22}$ Graph 2}
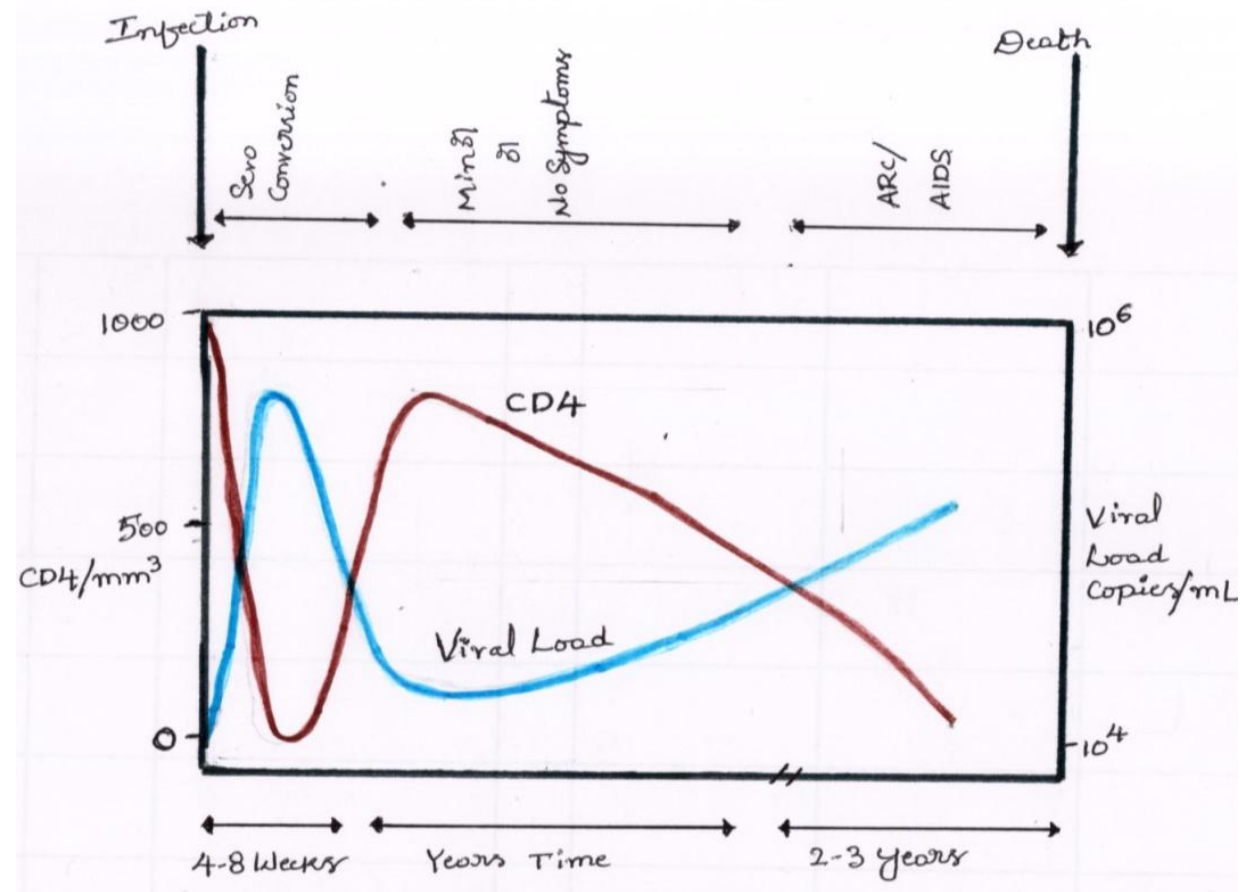

Seroconversion of HIV (Serological \& immunological progression) 


\section{Study Design \& Statistical Analysis}

Descriptive and frequency distribution was done using SPSS software Version 17.0 using Chisquare test and proportions and significance criteria of $\mathrm{P}<0.05$ is used in the analysis. Prevalence is calculated based on the no. of donations tested and no. of donations with positive results in screening tests.

\section{Limitations to study}

1. Genetic diagnostic aids are not used in screening in this study as in most parts of India and in the developing countries it is not in practice unlike for the Diagnosis of TB where Gene Xpert is used for CBNAAT (Catridge based nuclear acid amplification technique).

2. Certain parameters like separate screening for voluntary \& replacement donors is not done, nor for age and sex.

3. The blood donors are not screened even before donation but after donation only.

4. The sero-positive cases are not admitted for temporary isolation and treatment but simply advised them to consult a doctor. Blood transfusion practice should not be limited to transfusion of blood and finding out the complications only but must be extended to containing infected individuals till the course of treatment is over and the follow up period is over.

\section{Material and Methods}

In this retrospective study we aimed to estimate the seroprevalance of HIV, HBV, HCV and Syphillis among blood donars from a dedicated blood bank situated in north coastal Andhra in AP State in southern part of India. A very large sample of 17,632 blood bags screened data from Jan 2012 - Aug 2018 is collected obtaining permission from the secondary care district hospital of 250 beds at Rajahmahendravaram situated on NH5 that caters to about 55 lakh population. Blood was collected from healthy voluntary donars through camps/replacementvoluntary and a unique identification number was given to each donar. The data is analysed at the research centre, GIMSR at Visakhapatnam taking the help of the bio-statistician and the microbiologist. The blood group \& screening profile is tabulated and conclusions are drawn and comparison is made with similar studies in India and the west. Blood component study is not made separately but as part of other groups of blood as the screening methodology applied is same to all.

Lab Tests- Apart from routine blood tests, blood grouping is done and the screening procedure is initiated for HIV, HBsAg, HCV, Syphilis and Malaria. Though not routinely performed in India unlike in the west, the tests for HBV like $\mathrm{HBeAg}$, anti-HBe, anti-HBs, anti-HBc, IgM anti-HBc and HBV - DNA must be performed and also HCVAb, HCV-RNA. The relatively latest fourth generation Elisa reader and other equipment are used for screening HIV, HBsAg \& HCV. For syphilis RPR test is used and for Malaria the card test is used and not the QBC. Yet these tests are of limited sensitivity and specificity. The nucleic acid tests based on genetic markers are not yet widely used in India and other developing countries. The pathophysiology of seroconversion is better applied for the genetic based tests which are routinely used in the west.

\section{Screening Procedure}

After collection all samples were screened for Human Immunodeficiency Virus I \& II: By microwell ELISA to detect antibodies against HIV I \& II in plasma. Hepatitis B Virus: By microwell ELISA, Hepatitis C Virus: By microwell ELISA and Treponema Pallidum: Detection of Treponemal Antibodies (Reagin) by Rapid Plasma Reagin Test. Tests for HIV, HBsAg, HCV, Syphilis and Malaria are mandatory under drugs \& cosmetic act 1945 rules. 


\section{JMSCR Vol||06||Issue||11||Page 503-515||November}

\section{Diagnostic aids}

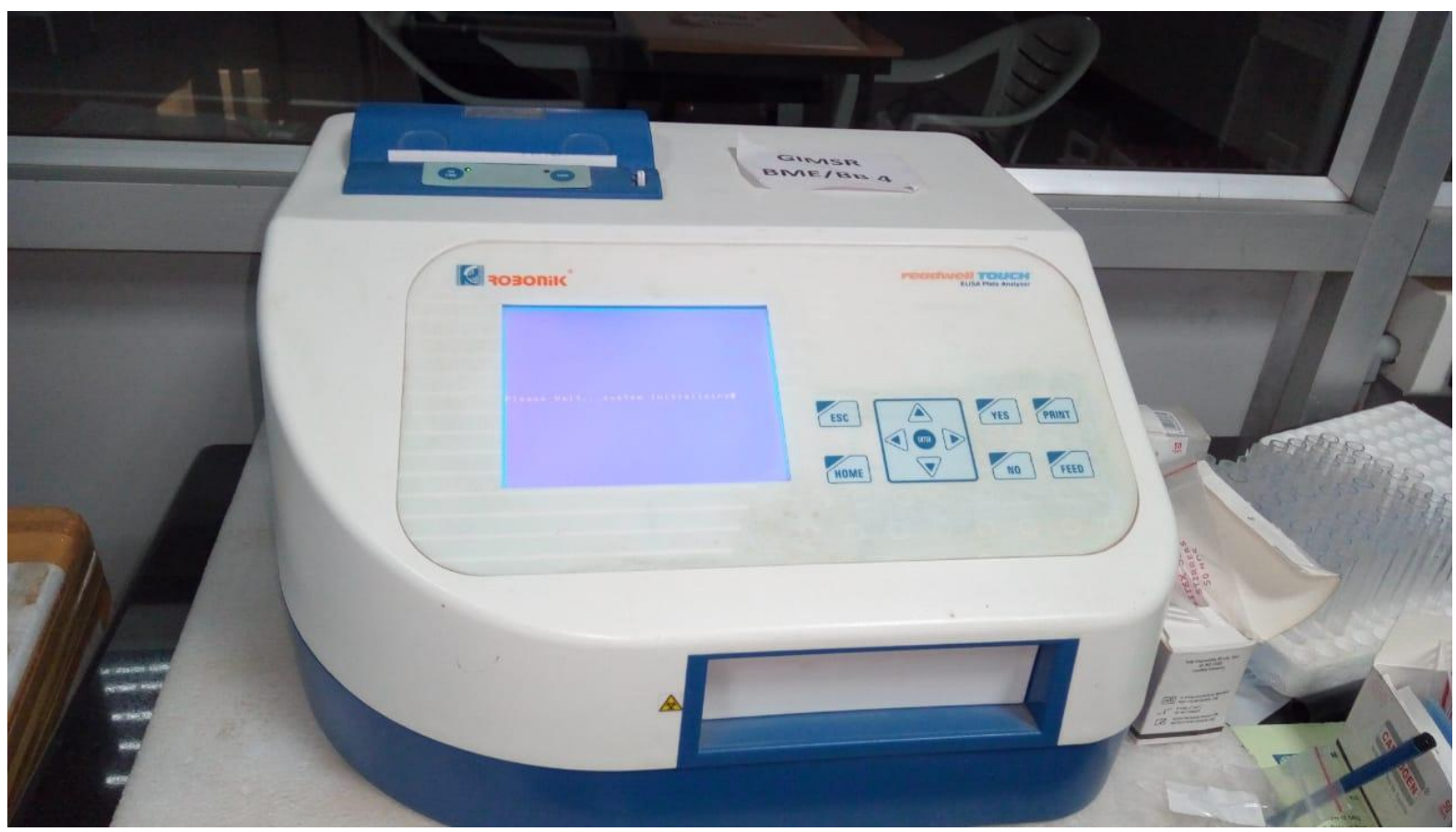

Microwell Elisa Reader

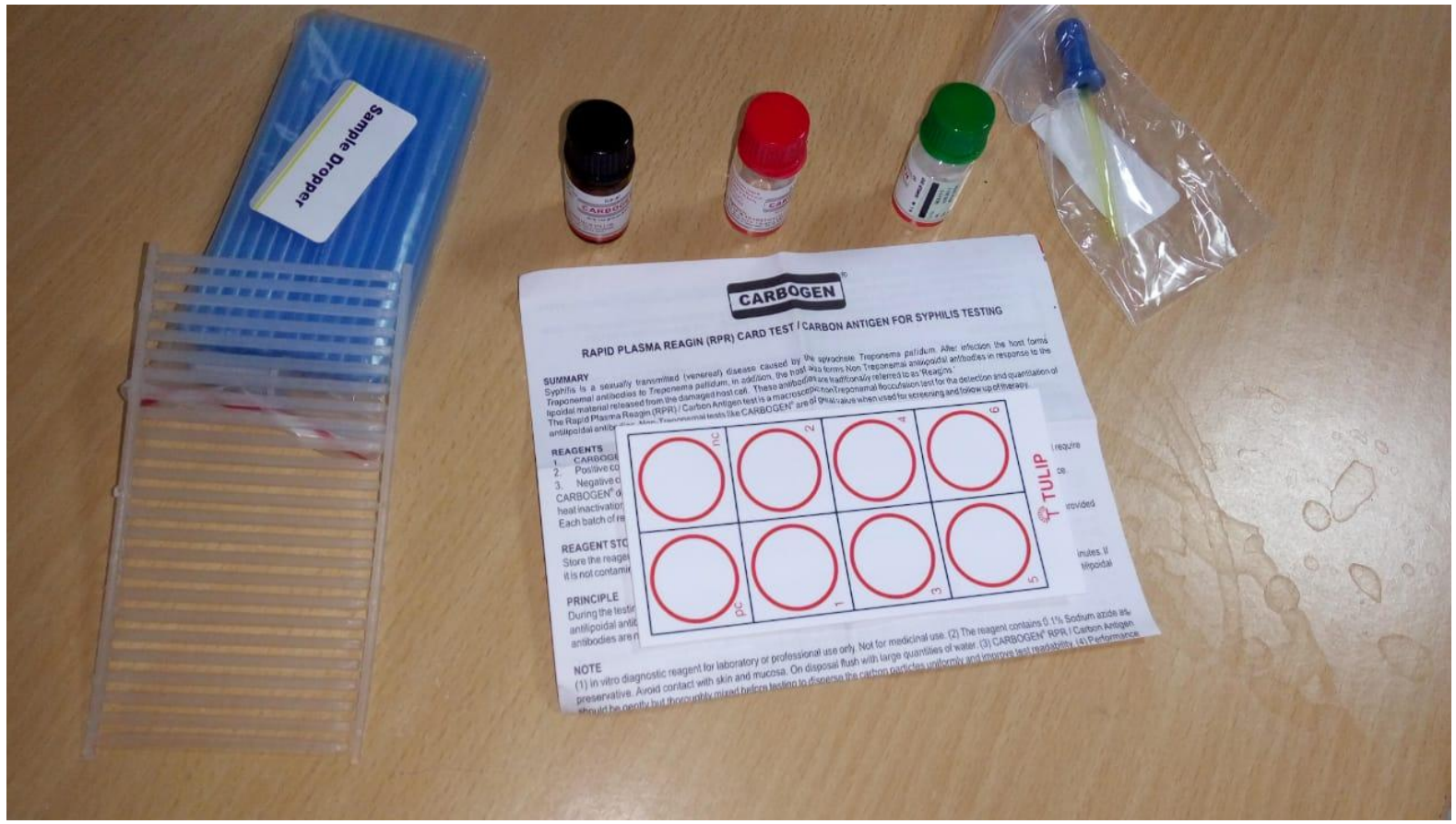

R P R - Test Kit for Syphilis

\section{Compiled Data}

Table - 1

\begin{tabular}{|l|c|c|c|c|c|c|c|}
\hline \multicolumn{8}{|l|}{ SERO POSITIVE PATTERN OF SCREENED BLOOD SAMPLES FROM DONAR BLOOD BAGS } \\
\hline Sero-positive & $\mathbf{2 0 1 2}$ & $\mathbf{2 0 1 3}$ & $\mathbf{2 0 1 4}$ & $\mathbf{2 0 1 5}$ & $\mathbf{2 0 1 6}$ & $\mathbf{2 0 1 7}$ & $\mathbf{2 0 1 8}$ \\
\hline HIV & 12 & 13 & 11 & 2 & 8 & 15 & 7 \\
\hline HbsAg & 36 & 30 & 18 & 45 & 63 & 65 & 40 \\
\hline HCV & 6 & 5 & 6 & 8 & 10 & 18 & 6 \\
\hline TOTAL & 54 & 48 & 35 & 55 & 81 & 98 & 53 \\
\hline
\end{tabular}


Table - 2

\begin{tabular}{|l|c|c|c|c|c|c|c|}
\hline NO. OF BLOOD BAGS COLLECTED (FROM 2012 - 8/2018) \\
\hline TYPE & $\mathbf{2 0 1 2}$ & $\mathbf{2 0 1 3}$ & $\mathbf{2 0 1 4}$ & $\mathbf{2 0 1 5}$ & $\mathbf{2 0 1 6}$ & $\mathbf{2 0 1 7}$ & Aug-18 \\
\hline VOLUNTARY & 1320 & 770 & 701 & 1357 & 1523 & 2061 & 1721 \\
\hline REPLACEMENT & 575 & 648 & 560 & 960 & 1883 & 2673 & 883 \\
\hline TOTAL & 1895 & 1418 & 1261 & 2317 & 3406 & 4734 & 2604 \\
\hline
\end{tabular}

Table - 3

\begin{tabular}{|c|c|c|c|c|}
\hline \multicolumn{6}{|c|}{ TOTAL BLEEDS AND SERO + VE PERCENTAGE - YEAR WISE } \\
\hline YEAR & $\begin{array}{c}\text { TOTAL } \\
\text { BLEEDS }\end{array}$ & ISSUES & $\begin{array}{c}\text { SERO } \\
+ \text { VE }\end{array}$ & PERCENTAGE \\
\hline $\mathbf{2 0 1 2}$ & 1895 & 1716 & 58 & 3.06 \\
\hline $\mathbf{2 0 1 3}$ & 1418 & 1291 & 49 & 3.45 \\
\hline $\mathbf{2 0 1 4}$ & 1261 & 1057 & 35 & 2.78 \\
\hline $\mathbf{2 0 1 5}$ & 2317 & 1954 & 55 & 2.37 \\
\hline $\mathbf{2 0 1 6}$ & 3406 & 3391 & 82 & 2.41 \\
\hline $\mathbf{2 0 1 7}$ & 4734 & 5490 & 98 & 2.07 \\
\hline Aug-18 & 2604 & 4250 & 53 & 2.03 \\
\hline TOTAL & 17635 & 19149 & 430 & 2.43 \\
\hline
\end{tabular}

Table - 4 :

\begin{tabular}{|c|c|c|c|}
\hline \multicolumn{4}{|c|}{$\begin{array}{l}\text { AVAILABLE BLOOD COMPONENT } \\
\text { STATISTICS }\end{array}$} \\
\hline COMPONENT & 2016 & 2017 & Aug-18 \\
\hline WB & 3150 & 3024 & 614 \\
\hline PRBC & 194 & 1256 & 1984 \\
\hline PCT & 39 & 88 & 10 \\
\hline FFP & 8 & 1122 & 1642 \\
\hline TOTAL : & 3391 & 5490 & 4250 \\
\hline
\end{tabular}

Table -5

SEROLOGICAL SCREENING OF BLOOD BAGS AND REACTIVITY PATTERN FROM 2012 - 2015

\begin{tabular}{|c|c|c|c|c|c|c|c|c|c|c|c|c|}
\hline \multirow[b]{2}{*}{ MONTH } & \multicolumn{3}{|c|}{ YEAR - 2012} & \multicolumn{3}{|c|}{ YEAR - 2013} & \multicolumn{3}{|c|}{ YEAR - 2014} & \multicolumn{3}{|c|}{ YEAR - 2015} \\
\hline & Bleedings & $\begin{array}{c}\text { sero - } \\
\text { type }\end{array}$ & $\begin{array}{c}\text { re } \\
\text { active }\end{array}$ & Bleedings & $\begin{array}{c}\text { sero - } \\
\text { type }\end{array}$ & $\begin{array}{c}\text { re } \\
\text { active }\end{array}$ & Bleedings & $\begin{array}{l}\text { sero - } \\
\text { type }\end{array}$ & $\begin{array}{c}\text { re } \\
\text { active }\end{array}$ & Bleedings & $\begin{array}{l}\text { sero - } \\
\text { type }\end{array}$ & $\begin{array}{c}\text { re } \\
\text { active }\end{array}$ \\
\hline \multirow{3}{*}{ JAN } & \multirow{3}{*}{187} & HIV & 1 & \multirow{3}{*}{61} & HIV & 0 & \multirow{3}{*}{155} & HIV & 2 & \multirow{3}{*}{212} & HIV & 0 \\
\hline & & $\mathrm{HbsAg}$ & 3 & & HbsAg & 3 & & HbsAg & 2 & & HbsAg & 3 \\
\hline & & $\mathrm{HCV}$ & 0 & & $\mathrm{HCV}$ & 0 & & $\mathrm{HCV}$ & 1 & & $\mathrm{HCV}$ & 0 \\
\hline \multirow{3}{*}{ FEB } & \multirow{3}{*}{130} & HIV & 1 & \multirow{3}{*}{84} & HIV & 1 & \multirow{3}{*}{71} & HIV & 1 & \multirow{3}{*}{64} & HIV & 0 \\
\hline & & HbsAg & 5 & & HbsAg & 1 & & HbsAg & 3 & & HbsAg & 3 \\
\hline & & $\mathrm{HCV}$ & 0 & & $\mathrm{HCV}$ & 0 & & $\mathrm{HCV}$ & 2 & & $\mathrm{HCV}$ & 0 \\
\hline \multirow{3}{*}{ MAR } & \multirow{3}{*}{167} & HIV & 2 & \multirow{3}{*}{132} & HIV & 1 & \multirow{3}{*}{143} & HIV & 1 & \multirow{3}{*}{119} & HIV & 0 \\
\hline & & HbsAg & 3 & & HbsAg & 4 & & HbsAg & 2 & & HbsAg & 3 \\
\hline & & $\mathrm{HCV}$ & 0 & & $\mathrm{HCV}$ & 0 & & $\mathrm{HCV}$ & 0 & & $\mathrm{HCV}$ & 1 \\
\hline \multirow{3}{*}{ APR } & \multirow{3}{*}{176} & HIV & 0 & \multirow{3}{*}{147} & HIV & 3 & \multirow{3}{*}{84} & HIV & 1 & & HIV & 0 \\
\hline & & HbsAg & 8 & & HbsAg & 5 & & HbsAg & 2 & 145 & HbsAg & 2 \\
\hline & & $\mathrm{HCV}$ & 2 & & $\mathrm{HCV}$ & 0 & & $\mathrm{HCV}$ & 1 & & $\mathrm{HCV}$ & 0 \\
\hline & & HIV & 1 & & HIV & 1 & & HIV & 1 & & HIV & 0 \\
\hline MAY & 113 & HbsAg & 6 & 64 & HbsAg & 1 & 61 & HbsAg & 0 & 163 & HbsAg & 8 \\
\hline & & $\mathrm{HCV}$ & 0 & & $\mathrm{HCV}$ & 0 & & $\mathrm{HCV}$ & 1 & & $\mathrm{HCV}$ & 3 \\
\hline & & HIV & 1 & & HIV & 0 & & HIV & 1 & & HIV & 1 \\
\hline JUN & 97 & HbsAg & 0 & 40 & HbsAg & 0 & 134 & HbsAg & 2 & 333 & HbsAg & 2 \\
\hline & & $\mathrm{HCV}$ & 1 & & $\mathrm{HCV}$ & 1 & & $\mathrm{HCV}$ & 0 & & $\mathrm{HCV}$ & 0 \\
\hline & & HIV & 2 & & HIV & 4 & & HIV & 1 & & HIV & 0 \\
\hline JUL & 185 & HbsAg & 4 & 219 & HbsAg & 5 & 129 & HbsAg & 1 & 200 & HbsAg & 6 \\
\hline & & $\mathrm{HCV}$ & 2 & & $\mathrm{HCV}$ & 1 & & $\mathrm{HCV}$ & 0 & & $\mathrm{HCV}$ & 1 \\
\hline & & HIV & 1 & & HIV & 1 & & HIV & 1 & & HIV & 0 \\
\hline AUG & 240 & $\mathrm{HbsAg}$ & 2 & 182 & HbsAg & 0 & 68 & $\mathrm{HbsAg}$ & 3 & 282 & $\mathrm{HbsAg}$ & 1 \\
\hline & & $\mathrm{HCV}$ & 1 & & $\mathrm{HCV}$ & 1 & & $\mathrm{HCV}$ & 0 & & $\mathrm{HCV}$ & 1 \\
\hline & & HIV & 0 & & HIV & 1 & & HIV & 1 & & HIV & 0 \\
\hline SEP & 198 & $\mathrm{HbsAg}$ & 1 & 179 & HbsAg & 5 & 116 & $\mathrm{HbsAg}$ & 1 & 159 & $\mathrm{HbsAg}$ & 2 \\
\hline & & $\mathrm{HCV}$ & 0 & & $\mathrm{HCV}$ & 0 & & $\mathrm{HCV}$ & 0 & & $\mathrm{HCV}$ & 0 \\
\hline & & HIV & 1 & & HIV & 1 & & HIV & 0 & & HIV & 0 \\
\hline OCT & 178 & HbsAg & 4 & 158 & HbsAg & 2 & 136 & HbsAg & 1 & 255 & HbsAg & 7 \\
\hline & & $\mathrm{HCV}$ & 0 & & $\mathrm{HCV}$ & 2 & & $\mathrm{HCV}$ & 1 & & $\mathrm{HCV}$ & 0 \\
\hline & & HIV & 0 & & HIV & 0 & & HIV & 1 & & HIV & 1 \\
\hline NOV & 133 & HbsAg & 0 & 73 & HbsAg & 4 & 88 & HbsAg & 1 & 185 & HbsAg & 1 \\
\hline & & $\mathrm{HCV}$ & 0 & & $\mathrm{HCV}$ & 0 & & $\mathrm{HCV}$ & 0 & & $\mathrm{HCV}$ & 2 \\
\hline & & HIV & 2 & & HIV & 0 & & HIV & 0 & & HIV & 0 \\
\hline DEC & 91 & HbsAg & 0 & 79 & HbsAg & 0 & 76 & HbsAg & 0 & 200 & HbsAg & 7 \\
\hline & & $\mathrm{HCV}$ & 0 & & $\mathrm{HCV}$ & 0 & & $\mathrm{HCV}$ & 0 & & $\mathrm{HCV}$ & 0 \\
\hline
\end{tabular}


Table - 6

\begin{tabular}{|c|c|c|c|c|c|c|c|c|c|}
\hline \multicolumn{10}{|c|}{ SEROLOGICAL SCREENING OF BLOOD BAGS AND REACTIVITY PATTERN FROM 2016 TO 8/2018 } \\
\hline \multirow[b]{2}{*}{ MONTH } & \multicolumn{3}{|c|}{ YEAR - 2016} & \multicolumn{3}{|c|}{ YEAR - 2017} & \multicolumn{3}{|c|}{ YEAR - 8/2018 } \\
\hline & Bleedings & $\begin{array}{c}\text { sero - } \\
\text { type }\end{array}$ & $\begin{array}{c}\text { re } \\
\text { active }\end{array}$ & Bleedings & $\begin{array}{c}\text { sero - } \\
\text { type }\end{array}$ & $\begin{array}{c}\text { re } \\
\text { active }\end{array}$ & Bleedings & $\begin{array}{c}\text { sero - } \\
\text { type }\end{array}$ & $\begin{array}{c}\text { re } \\
\text { active }\end{array}$ \\
\hline \multirow{3}{*}{ JAN } & \multirow{3}{*}{356} & HIV & 1 & \multirow{3}{*}{460} & HIV & 1 & \multirow{3}{*}{428} & HIV & 0 \\
\hline & & HbsAg & 7 & & HbsAg & 15 & & $\mathrm{HbsAg}$ & 6 \\
\hline & & $\mathrm{HCV}$ & 0 & & $\mathrm{HCV}$ & 4 & & $\mathrm{HCV}$ & 0 \\
\hline \multirow{3}{*}{ FEB } & \multirow{3}{*}{171} & HIV & 0 & \multirow{3}{*}{313} & HIV & 2 & \multirow{3}{*}{285} & HIV & 0 \\
\hline & & HbsAg & 3 & & HbsAg & 6 & & HbsAg & 3 \\
\hline & & $\mathrm{HCV}$ & 3 & & $\mathrm{HCV}$ & 1 & & $\mathrm{HCV}$ & 1 \\
\hline \multirow{3}{*}{ MAR } & \multirow{3}{*}{303} & HIV & 1 & \multirow{3}{*}{335} & HIV & 1 & \multirow{3}{*}{257} & HIV & 0 \\
\hline & & HbsAg & 10 & & HbsAg & 4 & & HbsAg & 4 \\
\hline & & $\mathrm{HCV}$ & 2 & & $\mathrm{HCV}$ & 2 & & $\mathrm{HCV}$ & 1 \\
\hline \multirow{3}{*}{ APR } & \multirow{3}{*}{207} & HIV & 0 & \multirow{3}{*}{337} & HIV & 1 & \multirow{3}{*}{326} & HIV & 4 \\
\hline & & HbsAg & 2 & & HbsAg & 6 & & HbsAg & 7 \\
\hline & & $\mathrm{HCV}$ & 0 & & $\mathrm{HCV}$ & 0 & & $\mathrm{HCV}$ & 1 \\
\hline \multirow{3}{*}{ MAY } & \multirow{3}{*}{238} & HIV & 1 & \multirow{3}{*}{485} & HIV & 0 & \multirow{3}{*}{407} & HIV & 0 \\
\hline & & HbsAg & 3 & & HbsAg & 3 & & HbsAg & 4 \\
\hline & & $\mathrm{HCV}$ & 1 & & $\mathrm{HCV}$ & 3 & & $\mathrm{HCV}$ & 2 \\
\hline & & HIV & 1 & & HIV & 1 & & HIV & 1 \\
\hline JUN & 294 & HbsAg & 7 & 331 & HbsAg & 7 & 207 & HbsAg & 4 \\
\hline & & $\mathrm{HCV}$ & 0 & & $\mathrm{HCV}$ & 3 & & $\mathrm{HCV}$ & 1 \\
\hline & & HIV & 1 & & HIV & 0 & & HIV & 2 \\
\hline JUL & 226 & HbsAg & 3 & 350 & HbsAg & 2 & 372 & HbsAg & 6 \\
\hline & & $\mathrm{HCV}$ & 0 & & $\mathrm{HCV}$ & 0 & & $\mathrm{HCV}$ & 0 \\
\hline & & HIV & 1 & & HIV & 1 & & HIV & 0 \\
\hline AUG & 389 & HbsAg & 4 & 397 & HbsAg & 3 & 322 & HbsAg & 6 \\
\hline & & $\mathrm{HCV}$ & 1 & & $\mathrm{HCV}$ & 0 & & $\mathrm{HCV}$ & 0 \\
\hline & & HIV & 2 & & HIV & 3 & & & \\
\hline SEP & 299 & HbsAg & 6 & 314 & HbsAg & 4 & & & \\
\hline & & $\mathrm{HCV}$ & 0 & & $\mathrm{HCV}$ & 2 & & & \\
\hline & & HIV & 0 & & HIV & 1 & & & \\
\hline OCT & 354 & HbsAg & 5 & 406 & HbsAg & 3 & & & \\
\hline & & $\mathrm{HCV}$ & 1 & & $\mathrm{HCV}$ & 3 & & & \\
\hline & & HIV & 0 & & HIV & 3 & & & \\
\hline NOV & 327 & HbsAg & 8 & 378 & HbsAg & 7 & & & \\
\hline & & $\mathrm{HCV}$ & 2 & & $\mathrm{HCV}$ & 0 & & & \\
\hline & & HIV & 0 & & HIV & 1 & & & \\
\hline DEC & 242 & HbsAg & 5 & 628 & $\mathrm{HbsAg}$ & 5 & & & \\
\hline & & $\mathrm{HCV}$ & 0 & & $\mathrm{HCV}$ & 0 & & & \\
\hline
\end{tabular}

Table - 7 :

\begin{tabular}{|l|c|c|}
\hline INFECTION & $\begin{array}{c}\text { NUMBER } \\
\text { OF } \\
\text { POSITIVES }\end{array}$ & $\begin{array}{c}\text { PERCEN } \\
\text { TAGE }\end{array}$ \\
\hline HIV & 68 & 0.38 \\
\hline HBSAg & 297 & 1.68 \\
\hline HCV & 59 & 0.33 \\
\hline SYPHILIS & 6 & 0.03 \\
\hline MALARIA & 0 & 0 \\
\hline
\end{tabular}

Table - 9:

\section{INDIAN AVERAGE OVERALL DONOR} TTI SEROPREVALENCE

$$
\begin{gathered}
\text { HIV }-0.3 \% \\
\text { HBV }-2-8 \% \\
\text { HCV }-2.0 \%
\end{gathered}
$$


Table -8

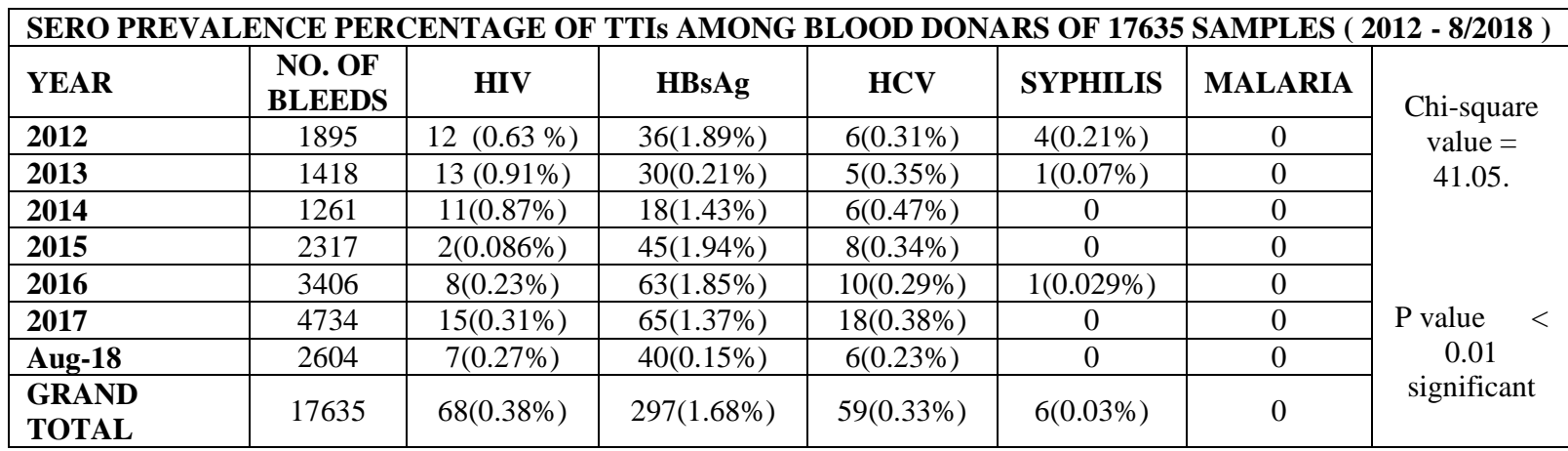

TOTAL SEROPOSITIVES OUT OF TOTAL BLEEDS : 17635/430 ( $2.44 \%$ )

\section{Other Studies for Comparison}

\section{Table - 10}

Other Study Similar to Present Study by Sample Size

YR 2009 TO 6/2016 STUDY OF SAMPLE SIZE 16,899, IJCMR, BY FULZELE ${ }^{2}$ PARAG PRABHAKAR,
MUMBAI
\begin{tabular}{|l|c|c|c|c|c|c|c|c|}
\hline YEARWISE DISTRIBUTION OF TTI \\
\hline YEAR & HIV & HBV & HCV & HIV \& HBV & HBV \& HCV & MP & VDRL & TOTAL \\
\hline $\mathbf{2 0 0 9}$ & 11 & 56 & 13 & 0 & 0 & 1 & 10 & 91 \\
\hline $\mathbf{2 0 1 0}$ & 20 & 40 & 13 & 0 & 0 & 1 & 5 & 79 \\
\hline $\mathbf{2 0 1 1}$ & 22 & 37 & 27 & 0 & 0 & 0 & 5 & 91 \\
\hline $\mathbf{2 0 1 2}$ & 10 & 40 & 10 & 0 & 1 & 1 & 3 & 65 \\
\hline $\mathbf{2 0 1 3}$ & 11 & 41 & 12 & 0 & 1 & 0 & 1 & 66 \\
\hline $\mathbf{2 0 1 4}$ & 11 & 40 & 17 & 0 & 0 & 0 & 3 & 71 \\
\hline $\mathbf{2 0 1 5}$ & 9 & 32 & 17 & 0 & 0 & 0 & 8 & 66 \\
\hline Jun-16 & 3 & 16 & 7 & 1 & 0 & 0 & 3 & 30 \\
\hline TOTAL & 97 & 302 & 116 & 1 & 2 & 3 & 38 & 559 \\
\hline
\end{tabular}

Table - 11

\begin{tabular}{|c|c|c|c|c|c|c|}
\hline \multicolumn{7}{|c|}{$\begin{array}{l}\text { SERO PREVALENCE OF HIV, HBSAg, HCV, SYPHILIS \& MALARIA IN DONORS } \\
\text { TOTAL OF 2,20,482 DONATIONS COLLECTED DURING JAN } 2008 \text { - DEC } 2014 \text { - STUDY OF AKANKSHA RAWAT, } \\
\text { DELHI }\end{array}$} \\
\hline YEAR & $\begin{array}{c}\text { TOTAL DONA } \\
\text { TIONS }\end{array}$ & HIV (\%) & HBsAg (\%) & HCV (\%) & SYPHILIS (\%) & MALARIA (\%) \\
\hline 2008 & 27,859 & $149(0.53 \%)$ & $478(1.71 \%)$ & $194(0.69 \%)$ & $814(2.92 \%)$ & $13(0.04 \%)$ \\
\hline 2009 & 29,790 & $101(0.33 \%)$ & $460(1.54 \%)$ & $177(0.59 \%)$ & $648(2.17 \%)$ & Nil \\
\hline 2010 & 32,553 & $97(0.29 \%)$ & $531(1.63 \%)$ & $221(0.67 \%)$ & $574(1.76 \%)$ & $16(0.04 \%)$ \\
\hline 2011 & 32,021 & $95(0.29 \%)$ & $505(1.57 \%)$ & $202(0.63 \%)$ & $464(1.44 \%)$ & $39(0.12 \%)$ \\
\hline 2012 & 32,902 & $96(0.29 \%)$ & $594(1.80 \%)$ & $266(0.80 \%)$ & $368(1.11 \%)$ & $34(0.10 \%)$ \\
\hline 2013 & 33,046 & $101(0.30 \%)$ & $482(1.45 \%)$ & $285(0.86 \%)$ & $392(1.18 \%)$ & $17(0.05 \%)$ \\
\hline 2014 & 32,311 & $81(0.25 \%)$ & $519(1.60 \%)$ & $268(0.82 \%)$ & $320(0.99 \%)$ & $21(0.06 \%)$ \\
\hline TOTAL & $2,20,482$ & $720(0.32 \%)$ & $3569(1.61 \%)$ & $1613(0.73 \%)$ & $3580(1.62 \%)$ & $140(0.06 \%)$ \\
\hline
\end{tabular}

Table - 12

\begin{tabular}{|c|c|c|c|c|c|c|}
\hline \multicolumn{7}{|c|}{ Seroprevalence of infectious markers from various Indian studies } \\
\hline & YEAR & HIV \% & HBsAg \% & $\begin{array}{c}\mathrm{HCV} \\
\%\end{array}$ & $\underset{\%}{\text { SYPHILIS }}$ & $\begin{array}{c}\text { MALARIA } \\
\%\end{array}$ \\
\hline Makroo et al ( New Delhi ) & 2015 & 0.24 & 1.18 & 0.43 & 0.23 & - \\
\hline Negi et al (Uttarakhand) & 2014 & 0.2 & 1.2 & 0.9 & 0.3 & 0.002 \\
\hline Arora et al (Haryana ) & 2010 & 0.3 & 17 & 1 & 0.9 & \\
\hline Pahuja et al ( Delhi ) & 2007 & 0.56 & 2.23 & 0.66 & - & \\
\hline Chandra T et al (Lucknow ) & 2009 & 0.23 & 1.96 & 0.85 & 0.001 & \\
\hline Srikrishna et al ( Karnataka ) & 2009 & 0.23 & 1.96 & 0.85 & 0.001 & \\
\hline Bhattacharya et al ( Kolkata ) & 2007 & 0.28 & 1.46 & 0.31 & 0.72 & \\
\hline Garg et al (Jodhpur, Rajashan ) & 2001 & 0.44 & 3.44 & 0.285 & 0.22 & \\
\hline Nirali Shah et al ( Gujrat ) & 2013 & 0.154 & 0.887 & 0.101 & 0.22 & \\
\hline Sharma et al ( Chandigarh ) & $1999-2002$ & $0.16-0.3$ & $1.55-0.99$ & 0.4 & 0.66 & \\
\hline Present study - KODANDA RAO et al (AP) & $2012-8 / 2018$ & 0.38 & 1.68 & 0.33 & 0.03 & 0.01 \\
\hline
\end{tabular}


Table -13

\begin{tabular}{|c|c|c|c|c|}
\hline \multicolumn{5}{|c|}{ INDIA WIDE STUDIES ON SEROPREVALENCE } \\
\hline AUTHOR & PLACE & YEAR & $\begin{array}{l}\text { SAMPLE } \\
\text { SIZE }\end{array}$ & $\begin{array}{l}\text { OVERALL SERO } \\
\text { PREVALENCE }\end{array}$ \\
\hline AKANKSHA RAWAT $^{4}$ et al & DELHI & Jan 20018 - Dec 2014 & $2,20,482$ & $4.36 \%$ \\
\hline NIRALI SHAH $^{3}$ & AHMEDABAD & Jan 2006 - July 2013 & 92,778 & $1.36 \%$ \\
\hline SWAPAN KUMAR SINHA $^{18}$ & KOLKATA & Jan 2007 - Dec 2008 & 44,173 & $5.80 \%$ \\
\hline FULZELE PARAG & MUMBAI & Jan 2009 - June 2016 & 16,899 & $4.10 \%$ \\
\hline RAJA SUNDARA MURTHY & MADURAI & Jan 2015 - Dec 2016 & 9,027 & $1.12 \%$ \\
\hline $\begin{array}{ll}\text { KODANDARAO } & \text { K. } \\
\text { (PRESENT STUDY ) } & \end{array}$ & $\begin{array}{l}\text { VISAKHAPATNA } \\
\text { M }\end{array}$ & Jan 20012 - Aug 2018 & 17,635 & $4.10 \%$ \\
\hline
\end{tabular}

FASOLA et al reported HIV prevalence of $13.2 \%$ in Nizeria

Table -14

\begin{tabular}{|c|c|c|c|c|c|}
\hline \multicolumn{6}{|c|}{ STUDIES ON HCV ${ }^{16}$ SERO PREVALENCE } \\
\hline $\begin{array}{ll}\text { PART } & \text { OF } \\
\text { INDIA } & \\
\end{array}$ & \multicolumn{2}{|c|}{ PERCENTAGE } & \multicolumn{2}{|c|}{$\begin{array}{c}\text { SOUTH INDIAN } \\
\text { AUTHORS }\end{array}$} & PERCENTAGE \\
\hline SOUTH INDIA & \multicolumn{2}{|r|}{$0.56 \%$} & \multicolumn{2}{|l|}{ SURESH et al } & $0.56 \%$ \\
\hline WEST INDIA & \multicolumn{2}{|r|}{$0.29 \%$} & \multicolumn{2}{|l|}{ MYTHREYE et al } & $0.22 \%$ \\
\hline EAST INDIA & \multicolumn{2}{|r|}{$0.35 \%$} & \multicolumn{2}{|c|}{ ARTHUR FATIMA $^{13}$ et al } & $0.01 \%$ \\
\hline NORTH INDIA & \multicolumn{2}{|r|}{$1.50 \%$} & \multicolumn{2}{|l|}{$\begin{array}{l}\text { RAJA SUNDARA } \\
\text { MURTHY et al }\end{array}$} & $0.56 \%$ \\
\hline \multicolumn{6}{|c|}{ Table - 15 : OTHER AUTHOR STUDIES ON HCV SERO PREVALENCE } \\
\hline \multicolumn{2}{|l|}{ AUTHOR } & YEAR & PLACE & & REVALENCE \\
\hline \multicolumn{2}{|c|}{ BATTACHARYA et al } & 2007 & WEST BENGAL & & $0.31 \%$ \\
\hline \multicolumn{2}{|l|}{ GUPTA et al } & 2004 & LUDHIANA & & $0.09 \%$ \\
\hline \multicolumn{2}{|l|}{ NIRALI SHAH et al } & 2013 & AHMADABAD & & $0.11 \%$ \\
\hline \multicolumn{2}{|l|}{ KODANDARAO } & 2018 & VISAKHAPATNAM & & $0.33 \%$ \\
\hline
\end{tabular}

\section{Results}

In the present study, HIV positivity has received a declining trend from 2012 to 2018, but HBsAg \& $\mathrm{HCV}$ show increasing trend as per table 1 . This may be due to increased awareness about HIV than HBsAg \& HCV. Yet the overall seroprevalence is also increasing over years. The multi mode transmission of $\mathrm{HBsAg} \& \mathrm{HCV}$ is also the reason.

Total of 430 sero positives are reported out of 17,635 samples and they were discarded unissued as per WHO guidelines. Total sero prevalence of $2.43 \%$ is reported in the present study which is similar to overall Indian sero prevalence of $2.4 \%$. Further HIV in $68(0.38 \%), \mathrm{HBsAg}$ in 297 (1.68 $\%)$ and $\mathrm{HCV}$ in $59(0.33 \%)$ samples reported and compared with general Indian donor population data of HIV ( $0.30 \%)$, HBV $(2-8 \%) 7 \mathrm{HCV}(2$ $\%)$. In the present study of southern part of India, $\mathrm{HCV}$ prevalence is less than the Indian average but other HIV and HBsAg are similar to Indian average as per Table $7 \& 9$ data.

\section{Discussion}

In the present study, HIV positivity has received a declining trend from 2012 to 2018, but HBsAg \& $\mathrm{HCV}$ show increasing trend as per table 1 . This may be due to increased awareness about HIV than HBsAg \& HCV. Yet the overall seroprevalence is also increasing over years. The multi mode transmission of $\mathrm{HBsAg} \& \mathrm{HCV}$ is also the reason. For all the tests the same Elisa reader is used. Hence the diagnostic aid matters little influencing the positivity. There is encouraging trend among Indian donor population towards donation as evidenced by increased number of blood bags noted in the table 2 data. But the replacement group outnumber the voluntary group among donations since 2016 but there is no corresponding trend in screened prevalence of TTI $^{12}$. Among blood components issued from 2016 to 8/2018, packed cell RBC and FFP (Fresh frozen plasma) show increased issue to patient's demands as per (table 4). The overall seropositivity has shown increased prevalence of 
3.06 to $3.45 \%$ above national average of $2.4 \%$ but from 2014 to Aug 2018 it maintained a plateau from 2.78 to $2.43 \%$ levelling national average (Table $7 \& 9$ ). This may be due to the use of latest screening diagnostic aids and the increased efficiency in screening (tables 3,5,6). Statistical methods : as per chi-square test the value is 41.05 and the $\mathrm{P}$ value is $<0.01$ (significant) when applied to all the data of 17,635 samples from 2012 to Aug 2018 out of which 430 are positive $(2.44 \%)$ as per table 8 . There is no significant increase of Syphilis \& Malaria.

\section{Comparision with Similar ${ }^{6,7,8}$ Studies: Fulzele et} al from Mumbai made similar study in 2016 with almost similar sample size of 16,899 (table 10) reported similar observations but co-infection of HBV with HIV and HCV were reported in his study. Swapan Kumar Sinha from Kolkata reported in 2008 (table 13) high overall sero prevalence of $5.80 \%$. But similar studies in the North eastern ${ }^{5}$ part at Gawhathi have not conformed to it.

Even the largest sample size of 2,20,482 by Akanksha Rawat et al, Delhi made similar observations in 2014 (table 11) except reporting high prevalence of syphilis than any such study (1.62 \%). Even Pahuja et al from Delhi in 2007 (table 12) reported high prevalence of HIV (0.56 $\%)$, HBsAg (2.23\%) which is more than what is reported by Makroo $^{10}$ et al in 2015, Negi et al from Uttarakhand, Arora et al from Haryana in 2010 and Chandra T et al from Lucknow in 2009, all are North Indian studies, and the present study by Kodandarao reported in 2018 from AP in South India, low prevalence of all parameters from sufficient data of 17,635 samples.

When it comes to HCV sero prevalence, as per the data in table $14 \& 15$ Suresh $^{14}$ et al from Tirupathi, South India and another study from North India showed similar trend of high prevalence of 0.56 $\%$. The least prevalence of $0.09 \%$ is reported by Gupta $^{11}$ et al from Luthiana but in 2004. The latest study by Kodandarao from Visakhapatnam in South India reported $0.33 \%$ of HCV prevalence which is far below the national average of $2.0 \%$.

\section{Conclusions}

HBV prevalence tops the list of total prevalence with fluctuating HIV and HCV data. Syphilis is reduced to the least due to wide use a potent bactericidal agent like Penicillin, which was very rampant $4-5$ decades back. Surprisingly transfusion Malaria is reported the least in all studies in India though there were several such cases treated all over India and developing countries in the past. CD 4 and VL are inversely proportional. In knowing the intensity of HIV disease both the values have to be correlated to initiate HAART therapy and blood transfusion. Better to perform all the battery of investigations including the antigenic and antibody profile of the viruses. Even before issuing blood bag for transfusion. That is the surest way to minimise TTi and to reduce sero prevalence to a great extent. Though not routinely performed in India unlike in the west, the tests for HBV like $\mathrm{HBeAg}$, anti-HBe, anti-HBs, anti-HBc, IgM anti-HBc and $\mathrm{HBV}$ - DNA must be performed and also HCV$\mathrm{Ab}, \mathrm{HCV}-\mathrm{RNA}$. This kind of sero response of $\mathrm{HBV}$ is clearly shown in the graph 2. Strict quality control, counselling of donars, elimination of paid repeated donors, using the latest time tested gadgets to screen the blood, avoiding contamination, training of blood bank lab technicians at regular intervals updating knowledge, maintaining regular uninterrupted power supply and avoiding resource crunch are very very essential for the best possible quality of blood transfusion. A qualified medical officer, nurse, lab technician in transfusion ${ }^{9}$ medicine or the clinical pathologist has to be posted in blood bank to render dedicated work. Paid, repeated and habituated donors must be eliminated from blood donation.

Future perspective: Nucleic acid amplification (N A T) techniques have to be used for screening like in the west. The anti $\mathrm{HCv}$ detected is confirmed by further detecting HCV - RNA. C L I $\mathrm{A}^{1}$ (Chemi luminescent immuno assay) is a fully automatic technique can be used for more sensitivity and specificity than even the latest generation of Elisa. Malaria (Pf/Pv) Ag test of 
genomix molecular diagnostics is preferred to detect even the lowest grade parasitaemia. Rapid immune-chromo graphic technique detects HRP2 antigen of pl. Falciparium and pLDH antigen of other pl. Species. Several other infections having blood affinity and their transmissible nature like CMV, EBV, etc., has to be kept in mind. Special diagnostic aids have to be devised and developed to detect them.

Thorough knowledge of seroconversion is of great value in screening and detecting the hidden load of TTi. The mutant nature of HBV is alarming and may not be detectable even by all advances in diagnostic serology.

Dreier's ${ }^{21}$ principles 2004 apply to detecting occult HBV or in window period donors who lack detectable HBsAg but positive for anti HBC and HBV DNA can be screened and are of great value in minimising the sero prevalent burden in the community. The mutant strains of HBV poses a grave problem and so the burden of HBV is still significant in the community despite advances in the field of immunology and molecular biology.

HBV-DNA can be measured by PCR (polymerase chain reaction). Viral loads are use only in excess of $10^{5}$ copies per $\mathrm{ml}$ in the presence of active viral replication, important in identifying patients with pre-core mutants. Specific HBV genotypes of A $\&$ B which cause more aggressive disease can be identified using PCR and plan treatment with interferons. HbeAg reflects active replication 5 $10 \% \mathrm{HBV}$ only develop chronic infection and 90 $\%$ may clear the virus. Yet $90 \%$ of mother to child transmission results in chronic infection. Hence the focus must be on this particular age and sex population.

\section{Financial support: Nil \\ Conflict of interest: Nil}

\section{Acknowledgement}

We are grateful to the NACO/APSACS incharge at the hospital in issuing the data and encouraging research. The integrated approach involving the Prof. microbiologist, the clinical pathologist, the bio statistician and the data entry operator cum the junior assistant Mr. Nammi Srinivasa Rao at GITAM Institute of medical sciences and research, Hospital, at Visakhapatnam, AP is indeed praiseworthy.

\section{References}

1. Raja sundaramurthy, Ramesh Arunagiri Seroprevalence of transfusion transmitssible infections among blood donars by chemiluminescent assay in a tertiary care center - J Infect Dev Ctries 2018: 12(1):031-036. Doi:10.3855/jidc.9430

2. Fulzele Parag Prabhakar, Yasmeen Khatib - Infectious Disease Screening and Trends in Blood Donors in a Hospital based Blood Bank in Mumbai, Western India International Journal of Contemporary Medical Research Volume 4 Issue 2 | February 2017

3. Dr. Nirali Shah, Dr. J.M. Shah - Sero prevalence of HBV, HCV, HIV and syphilis among blood donors at a tertiary Care Teaching Hospital in Western India GUJARAT MEDICAL JOURNAL / DECEMBER - 2013 Vol. 68 No. 2

4. Akanksha Rawat, Preeti Diwakar Seroprevalence \& changing trends of transfusion-transmitted infections amongst blood donors in a Regional Blood Transfusion Centre in north india - Indian J Med Res 146, November 2017, pp 642645

5. Ritapa Ghosh and Naba Kumar Hazarika Seroprevalence of HBV and HCV among Healthy Blood Donors in a Tertiary Care Hospital of Northeast India : a 5-year Retrospective study - International Journal of Current Microbiology and Applied Sciences Volume 7 Number 07 (2018)

6. Shalini Sunderam, Shilpa Karir - SeroPrevalence of Transfusion Transmitted Infections among Blood Donors at Blood Bank of Rajendra Institute of Medical Sciences, Ranchi - Healthline Journal Volume 6 Issue 1 (January - June 2015) 
7. Bhagwan Singh Yadav, Amit V Varma Seroprevalence of transfusion-transmitted infections (TTIs) in blood donors: a study from central India - International Journal of Medical Science and Public Health | 2016 | Vol 5 | Issue 06 - International Journal of Medical Science and Public Health | 2016 | Vol 5 | Issue 06

8. Nagababu Pyadala, Soumendra Nath Maity - Seroprevalence of Transfusion Transmissible Infections among Blood Donors attending in a Rural Teaching Hospital at Sangareddy - Int J Biol Med Res.2015;6(4):5249-5253

9. Bihl F, Castelli D, Marincola F, Dodd RY, Brander C (2007) Transfusion-transmitted infections. J Transl Med 5: 25.

10. Makroo RN, Walia RS, Chowdhry M, Bhatia A, Hegde V, Rosamma NL (2013) Seroprevalence of anti-HCV antibodies among blood donors of north India. Indian J Med Res.138:125-128.

11. Sharma DC, Rai S, Bharat S, Iyenger $S$, Gupta S, Jain B (2014) A 10 years comparative study to assess trends in seroprevalence of transfusion transmitted infections among blood donors at Gwalior, India. Open J Blood Dis 4: 24-32 5. Lindholm PF, Annen K, Ramsey G (2011).

12. Mandal R, Mondal K (2016) Transfusion transmissible infections among blood donors from a sub-Himalayan rural tertiary care centre in Darjeeling, India. J Tradit Complement Med 6: 224-229.

13. Fatima A, Begum F, Kumar K (2016) Seroprevalence of transfusion transmissible infections among blood donors in Nizamabad district of Telangana State. A six years study. IAIM 3: 73-78.

14. Suresh B, Sreedhar Babu KV, Venkataramana B V, Chandra Mouli P (2016) Burden of transfusion transmissible viral infections among blood donors at a tertiary care referral teaching hospital in South India. J Clin Sci Res 5: 160-163.
15. WHO Hep B fact sheet, July 2017 \& WHO Hep C fact sheet, October 2017.

16. Rahman S, Gogoi G. Seroprevalence of Hepatitis B and Hepatitis C virus infection among healthy blood donors in a tertiary care hospital: A retrospective study. Global Journal for Research Analysis. 2016 Mar; 5 (3): 326-328.

17. Bommanahalli B, Javali R, CM MS, Gouda K, Siddartha K, Shashikala KP. Seroprevalence of hepatitis B and hepatitis $\mathrm{C}$ viral infections among blood donors of central Karnataka, India. Int J Med Sci Public Health 2014;3(1):272-5.

18. Swapan Kumar Sinha, Sudarshana Roychoudhury - Prevalence of HIV, Hepatitis B, Hepatitis C and Syphilis in donor's blood : A study from eastern part of India - Open Journal of hematology, 2012, 3-1.

19. Shukla RS, Bhuyan KK. Can Data on HIV Sero-reactivity among Blood Donor's Provide an Insight into HIV Prevalence in the General population?. Indian J Public Health. 2007; 51: 14-21.

20. Arora D, Arora B, Khetarpal A. Seroprevalence of HIV, HBV, HCV and Syphilis in blood donors in southern Haryana, Indian J Pathol Mocrobiol. 2010;53: 308-9.

21. Dreier J L et al - low level viraemia of Hepatitis 'B" Virus in a anti $\mathrm{HBc}$ and anti HBs positive blood donors - transfusion medicine - 2004; 14; 97-103.

22. Davidson's principles \& practice of medicine, $21^{\text {st }}$ edition - pages 389,394 , 949,950,951. 